\title{
Obesity at Conception Programs the Opioid System in the Offspring Brain
}

\author{
Nicola M Grissom ${ }^{1,2}$, Randolph Lyde', Lori Christ ${ }^{3}$, Isaac E Sasson ${ }^{4}$, JesseLea Carlin ${ }^{1,2}$, Alexa P Vitins ${ }^{4}$, \\ Rebecca A Simmons ${ }^{2,3,5}$ and Teresa M Reyes*, ${ }^{*, 2}$ \\ 'Department of Pharmacology, Perelman School of Medicine at the University of Pennsylvania, Philadelphia, PA, USA; ${ }^{2}$ Institute for Translational \\ Medicine and Therapeutics, Perelman School of Medicine at the University of Pennsylvania, Philadelphia, PA, USA; ${ }^{3}$ Department of Pediatrics, \\ Children's Hospital of Philadelphia, Philadelphia, PA, USA; ${ }^{4}$ Department of Obstetric and Gynecology, Perelman School of Medicine at the \\ University of Pennsylvania, Philadelphia, PA, USA; ${ }^{5}$ Department of Pediatrics, Perelman School of Medicine at the University of Pennsylvania, \\ Philadelphia, PA, USA
}

\begin{abstract}
Maternal obesity during pregnancy increases the risk for offspring obesity, in part through effects on the developing brain. Previous research has shown that perinatal consumption of highly palatable foods by the mother can influence the development of offspring taste preferences and alter gene expression within the central nervous system (CNS) reward system. Opioids stimulate consumption of both fats and carbohydrates, and overconsumption of these energy dense foods increases the risk for obesity. What has remained unclear is whether this risk can be transmitted to the offspring before gestation or if it is wholly the gestational exposure that affects offspring brain development. Utilizing an embryo transfer experimental design, 2-cell embryos were obtained from obese or control dams, and transferred to obese or control gestational carriers. Expression of the mu-opioid receptor (MOR), preproenkephalin (PENK), and the dopamine transporter was evaluated in the hypothalamus and reward circuitry (ventral tegmental area, prefrontal cortex, and nucleus accumbens) in adult and late embryonic brains. Obesity before pregnancy altered expression levels of both MOR and PENK, with males relatively more affected than females. These data are the first to demonstrate that obesity at conception, in addition to during gestation, can program the brain reward system.
\end{abstract}

Neuropsychopharmacology (2014) 39, $801-810$; doi:I0.1038/npp.2013.193; published online 9 October 2013

Keywords: Mu opioid receptor; preproenkephalin; obesity; high-fat diet; pregnancy; reward

\section{INTRODUCTION}

The incidence of obesity among pregnant women in the United States ranges from 20 to 38\% (Yogev and Catalano, 2009). Maternal obesity increases the lifetime risk for development of chronic diseases in the offspring, including obesity (Yu et al, 2011) and adverse neurobehavioral outcomes (Lieshout et al, 2011), including autism spectrum disorders (Dodds et al, 2011; Krakowiak et al, 2012), attention deficit-hyperactivity disorder (ADHD) (Ray et al, 2009; Rodriguez, 2010), anxiety/depression (Alati et al, 2009), difficulty with emotional regulation (Rodriguez, 2010), and cognitive delay (Hinkle et al, 2012). Animal models of maternal obesity and/or high-fat diet (HFD) consumption have replicated similar findings, including anxiety (Bilbo and Tsang, 2010; Sullivan et al, 2010), spatial learning deficits (Bilbo and Tsang, 2010), altered response

\footnotetext{
*Correspondence: Dr TM Reyes, Institute for Translational Medicine and Therapeutics, University of Pennsylvania, 10-131 Smilow Center For Translational Research, 3400 Civic Center Boulevard, Building 421, Philadelphia, PA 19104, USA, Tel: + 215573 2991, Fax: + 1215 573 2236, E-mail: reyestm@mail.med.upenn.edu

Received 9 April 2013; revised 29 July 2013; accepted 29 July 2013; accepted article preview online 8 August 2013
}

to drugs of abuse (Naef et al, 2011; Shalev et al, 2010), decreased hippocampal dendritic arborization (Tozuka et al, 2010), and alterations in the central nervous system (CNS) gene expression (Bilbo and Tsang, 2010; Chang et al, 2008; Gupta et al, 2009; Sullivan et al, 2010; Vucetic et al, 2010). Given these broad effects, it is critical to better understand how maternal obesity around the time of pregnancy affects offspring brain development.

The central reward system is vulnerable to maternal obesity and/or maternal consumption of an HFD during pregnancy. Adult offspring exposed to high-fat or high-sugar diets during pregnancy and/or lactation show an increased preference for high-fat or high-sugar foods (Bayol et al, 2007; Ong and Muhlhausler, 2011; Teegarden et al, 2009; Vucetic et al, 2010). Alterations in the expression of both dopamine and opioid-related genes have been documented in offspring exposed to high-fat and/or high-sugar diets perinatally (Naef et al, 2011; Ong and Muhlhausler, 2011; Teegarden et al, 2009; Vucetic et al, 2010). Dopamine, opioids, and rewardrelated behavior are associated with not only obesity, but also numerous adverse mental health outcomes (ADHD, depression, and addiction).

The $\mu$-opioid receptor (MOR) is a central player in coding the rewarding properties of natural stimuli such as palatable 
foods (Shin et al, 2010), driving hedonically driven feeding processes, as well as opiate drugs (eg, morphine and heroin; Klein et al, 2009) and nicotine (Berrendero et al, 2010). Activation of the MOR in the ventral striatum drives animals to selectively seek out fat-containing foods (Zhang and Kelley, 2000), whereas activation of MOR in the cortex drives the selective consumption of high-carbohydrate foods (Mena et al, 2011). Importantly, a recent study has confirmed the relationship between MOR and fat intake in human subjects (Haghighi et al, 2013). Therefore, increased signaling through the MOR may promote obesity through the selective enhancement of consumption of highly palatable, energy dense (high fat and high carbohydrate) foods. Indeed, MOR is a target for a potential therapeutic directed toward obesity and disordered eating (Nathan et al, 2012).

Exposure to an HFD and/or obesity during pregnancy can alter the central opioid system. What remains unclear is whether risk can originate before pregnancy, or whether gestation represents the key period of vulnerability. This information would be critical for women planning pregnancies, and the physicians who counsel them, to ensure the optimal health and development of the baby. To determine whether obesity before pregnancy could program adverse fetal brain development, an embryo transfer experiment was completed in which 2-cell embryos were taken from lean or obese dams, and transferred into lean or obese gestational carriers.

\section{MATERIALS AND METHODS}

\section{Animals and Diet}

All experimental procedures were approved by the Institutional Animal Care and Use Committee review boards of the Children's Hospital of Philadelphia and/or the University of Pennsylvania. Female C57BL/6J mice (Jacksons Labs) and CF-1 (Charles River) at 4 weeks of age were housed four animals per cage and given free access to feed and water. Two isocaloric diets were used: control diet (Lab Diet 5015) and an HFD (Harlan TD.06414). The control diet is composed of $11 \%$ fat by weight, and provides $4.7 \mathrm{kcal} / \mathrm{g}$ with calories provided by $19.8 \%$ protein, $45.9 \%$ carbohydrates $(3.8 \%$ sugar), and $25.3 \%$ fat. The HFD is composed of $34 \%$ fat by weight, and provides $5.1 \mathrm{kcal} / \mathrm{g}$ with calories provided by $18.4 \%$ protein, $21.3 \%$ carbohydrates (9.0\% sugar), and $60.3 \%$ fat. Female animals were permitted to feed ad libitum for at least 12 weeks before mating. Male C57BL/6J mice and transgenic C57BL/6$\mathrm{Tg}$ (UBC-GFP)30Scha/J (Jackson Labs, stock \#4353) were between 6 and 18 weeks of age and were maintained on the control diet and used for breeding until their weight exceeded $34 \mathrm{~g}$.

\section{Embryo Transfer}

Control or HFD female C57BL/6J mice were utilized as embryo donors and control or HFD female CF1 mice were utilized as gestational carriers/embryo recipients. Donors were age matched, and HFD donors weighed at least $28 \mathrm{~g}$. Control and HFD embryo donors were superovulated with an intraperitoneal injection of $5 \mathrm{IU}$ pregnant mare's serum gonadotropin and $46 \mathrm{~h}$ later with a similar injection of $5 \mathrm{IU}$ human chorionic gonadotropin (Sigma). HFD dams were mated with wild-type C57BL/6J males. Control dams were mated with C57BL/6-Tg(UBC-GFP)30Scha/J males, which carry a transgene that contains the GFP coding region under the control of the human ubiquitin $\mathrm{C}$ promoter (Schaefer, 2001). This paradigm permits transfer of both the HFDderived embryos and the control-derived embryos into the same recipient minimizing the effects of the variability between recipients on the two donor groups.

Twenty-four hours after detection of a vaginal plug, donor females were killed, and 2-cell embryos were harvested from the ampullary region of the fallopian tube and collected into M2 medium (Millipore) using standard techniques (Nagy et al, 2003; Shirley et al, 1986; Wang and Dey, 2006). Embryos from similar groups were pooled and incubated at $37^{\circ} \mathrm{C}$ for $1 \mathrm{~h}$ before transfer into pseudopregnant $\mathrm{CF} 1$ females, which had been maintained on either the control or HFD. These pseudopregnant CF1 females had been primed for embryo transfer by naturally mating with vasectomized males the evening before transfer (Champlin et al, 1973). For each recipient, 10 HFD-derived and 10 control-derived embryos were selected from their respective pools, mixed together, and 10 randomly selected embryos were surgically transferred into each oviduct of the recipient. The day of embryo transfer was considered as embryonic day 0.5 post conception. Pregnancies were allowed to proceed until e17.5, or to delivery. Sex determination for the e17.5 embryos was performed by PCR amplification of male-specific Sry gene. Placenta DNA was amplified with Sry forward primer $5^{\prime}$-TTGTCTAGAGAGCATGGAGGGCCATGTCAA- $3^{\prime}$ and reverse primer $5^{\prime}$-C CACTCCTCTGTGACACTTTAGCCCTCCGA-3'. Reactions were performed in $25 \mu \mathrm{l}$ for 26 cycles $\left(95^{\circ} \mathrm{C} 30 \mathrm{~s}, 60^{\circ} \mathrm{C}\right.$ $1 \mathrm{~min}$, and $72{ }^{\circ} \mathrm{C} 30 \mathrm{~s}$ ). $\beta$-Actin was used as a control for amplification in each reaction.

Recipients were maintained on their prepregnancy diet after embryo transfer. For the group of recipients that delivered live progeny, they were continued on the control or HFD through lactation. Six control and five HFD recipients delivered live-born pups. Mean litter size was eight pups (range 6-12), and the number of GFP $+v s$ GFP - pups did not differ within a litter. At e17.5, pups from all experimental groups weighed significantly less than the control pups. At 14 weeks of age (adult), both male and female pups exposed to HFD during gestation weighed significantly more than controls or those animals exposed to HFD only pregestation (Supplementary Table S1) (Sasson et al, submitted). After weaning at 3 weeks of life, all pups were placed on the control diet and housed at four animals per cage through 14 weeks of life, at which point they were euthanized.

\section{Genomic DNA and Total RNA Isolation}

Animals were euthanized with an overdose of carbon dioxide, followed by cervical dislocation; a method recommended by the Panel on Euthanasia of the American Veterinary Medical Association. Brains were then rapidly removed and placed in RNAlater (Ambion, Austin, TX, USA) for $24 \mathrm{~h}$ at $4{ }^{\circ} \mathrm{C}$ before dissection. Brain dissections to isolate the prefrontal cortex, the nucleus accumbens, 
Table I Analysis of Gene Expression Results in Adult Male and Female Brains

\begin{tabular}{|c|c|c|c|c|c|c|c|}
\hline & Interaction & Pregestation & Gestation & & Interaction & Pregestation & Gestation \\
\hline \multicolumn{8}{|l|}{ Males } \\
\hline$N A c$ & & & & HYP & & & \\
\hline \multirow[t]{2}{*}{ MOR } & - & $F(I, \mid 8)=6.05$ & $F(I, 18)=9.9$ & MOR & $F(I, 19)=9.0$ & - & $F(1,19)=8.62$ \\
\hline & & 0.024 & 0.0056 & & 0.0074 & & 0.0085 \\
\hline PENK & - & - & - & PENK & - & - & $F(1,19)=11.03$ \\
\hline \multirow[t]{2}{*}{ MOR } & $F(1,19)=11.65$ & - & $F(1,19)=11.7$ & MOR & $F(1,19)=8.67$ & $F(1,19)=9.36$ & $F(1,19)=8.27$ \\
\hline & 0.0029 & & 0.0029 & & 0.0074 & 0.0064 & 0.0097 \\
\hline \multirow[t]{2}{*}{ PENK } & $F(1,17)=11.12$ & - & $F(I, 17)=9.98$ & PENK & - & - & - \\
\hline & 0.0039 & & 0.0057 & & & & \\
\hline \multirow[t]{2}{*}{ MOR } & - & $F(1,19)=6.24$ & $F(I, 19)=30.38$ & MOR & - & - & $F(1,19)=61.08$ \\
\hline & & 0.022 & $<0.000$ & & & & $<0.000 \mid$ \\
\hline \multirow[t]{2}{*}{ PENK } & - & $F(I, 19)=4.93$ & $F(1,19)=16.28$ & PENK & - & - & $F(I, 19)=5.08$ \\
\hline & & 0.039 & 0.0007 & & & & 0.036 \\
\hline PFC & VTA & & & & & & \\
\hline \multirow[t]{2}{*}{ MOR } & - & - & $F(1,19)=31.04$ & MOR & - & - & $F(1,19)=10.82$ \\
\hline & & & $<0.000$ & & & & 0.0039 \\
\hline \multirow[t]{3}{*}{ PENK } & - & - & $F(1,19)=5.58$ & PENK & - & - & $F(I, I 6)=8.21$ \\
\hline & & & 0.029 & & & & 0.0012 \\
\hline & & & & DAT & - & - & $F(1,17)=11.12$ \\
\hline
\end{tabular}

ventral tegmental area, and hypothalamus were preformed as previously described (Vucetic et al, 2010). Total RNA was isolated using the AllPrep DNA/RNA Mini Kit (Qiagen).

\section{Gene Expression Analysis by Quantitative Real-Time PCR}

For each individual sample, 500 ng of total RNA was used in reverse transcription using the High Capacity Reverse Transcription Kit (ABI, Foster City, CA, USA). Expression of target genes was determined by quantitative RT-PCR using gene-specific Taqman Probes with Taqman gene expression Master Mix (ABI) on the ABI7900HT Real-Time PCR Cycler. Gene probes are listed in Supplementary information. Relative amount of each transcript was determined using delta CT values as previously described in Pfaffl (2010). Changes in gene expression were calculated against an unchanged glyceraldehyde 3-phosphate dehydrogenase (GAPDH) standard. Primers used are GAPDH Mm99999915_g1, preproenkephalin (PENK) Mm01212875_m1, opioid receptor, mu 1 (MOR) Mm01188089_m1, and solute carrier family 6 (neurotransmitter transporter, dopamine) member 3 (DAT) Mm00438388_m1.

\section{Methylated DNA Immunoprecipitation Assay}

MOR promoter methylation was evaluated in the PFC samples from adult male and female samples. Methylated DNA immunoprecipitation (MeDIP) assay was performed using the MagMeDIP kit (Diagenode, Denville, NJ, USA). Methylated DNA was immunoprecipitated using $0.15 \mu \mathrm{l}$ of magnetic beads coated with anti-5-methylcytidine antibody (Diagenode) or mouse preimmune serum. Quantity of DNA used was $833.3 \mu \mathrm{g}$ for the IP and $83.3 \mu \mathrm{g}$ for the input. Enrichment in MeDIP fraction was determined by quantitative RT-PCR using ChIP-qPCR Assay Master Mix (SuperArray) on the ABI7900HT Real-Time Cycler. For all genes examined, primers were obtained from SuperArray (ChIPqPCR Assays ( - 01) kb tile, SuperArray) for the amplification of genomic regions spanning the $\mathrm{CpG}$ sites located $\sim 300-500 \mathrm{bp}$ upstream of the transcription start sites. MeDIP results were expressed as fold enrichment of immunoprecipitated DNA for each site. To calculate differential occupancy fold change (\% enrichment), the MeDIP DNA fraction CT values were normalized to input DNA fraction CT values. Primers used are OPRM1 Mouse Oprm1, NM_001039652.1 ( - )01 kb: GPM1042701( - )01A, ACTIN Mouse Actb, NM_007393.2 ( - )01Kb: GPM1051747 

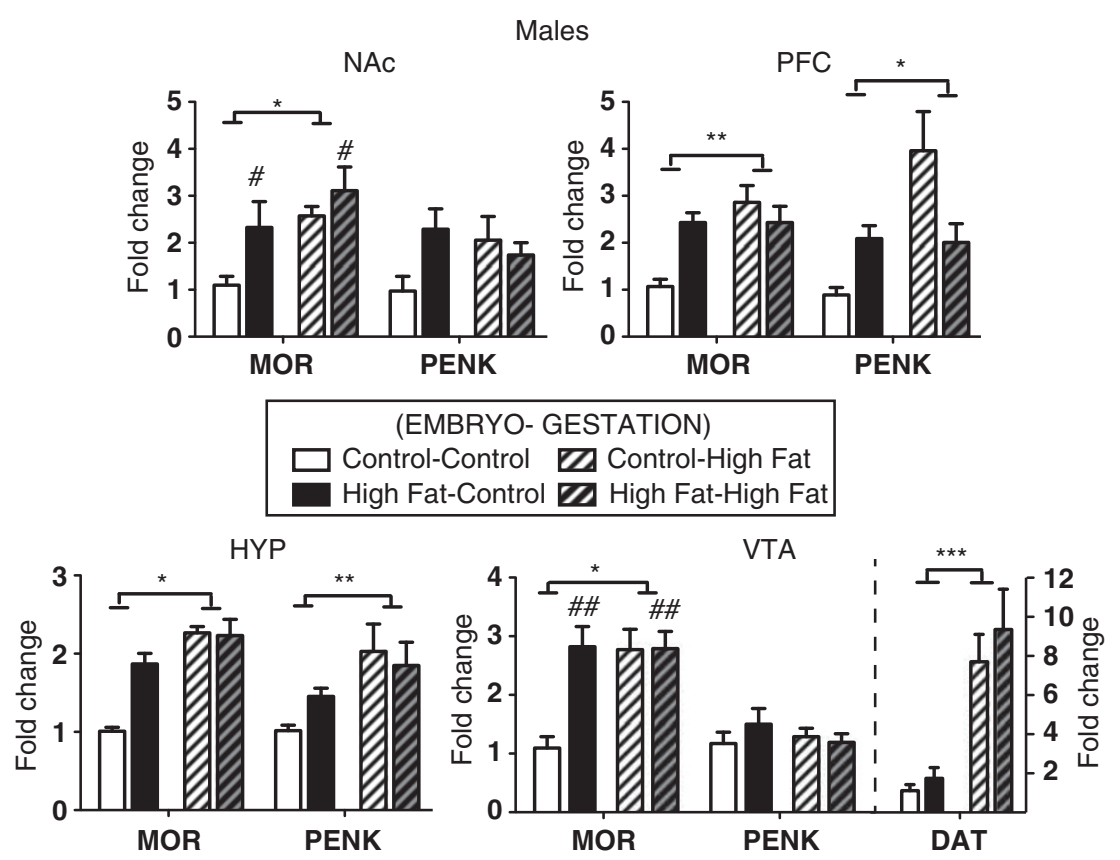

Figure I Gene expression in the adult male brain. mRNA for MOR and PENK was evaluated in the NAc, PFC, HYP, and VTA. DAT was also measured in the VTA. Main effect for prepregnancy obesity designated as ${ }^{\#} P<0.05,{ }^{\#} P<0.01$. Brackets indicate main effect for gestational obesity $(* P<0.01$, *** $P<0.005$, **** $P<0.00$ I).

( - )01A, SLC6A3 Mouse Slc6a3, NM_010020.3 ( - )01 Kb: GPM1031009( - )01A.

\section{Statistics}

Two-way ANOVA was used to determine the effect of obesity, either prepregnancy or during gestation, on mRNA expression in the offspring brain. Significant interactions between the two factors were followed by one-way ANOVAs to examine differences between the groups. $P<0.05$ was considered as significant.

\section{RESULTS}

MOR and PENK mRNA was evaluated within rewardrelated circuitry in the brain (VTA, NAc, and PFC), and hypothalamus of male and female adult offspring. Two-way ANOVA was used to evaluate the relative and/or combined effect of prepregnancy obesity (main effect for embryo donor) $v s$ obesity during gestation/lactation (main effect for gestational environment). Table 1 summarizes the results and statistical analyses; for clarity, only main effects are indicated in the figures. For male offspring, the pattern of responses was similar for MOR in the VTA, PFC, and HYP, with a significant interaction between embryo donor and gestation $(\mathrm{F}(1,19)=8.97, P=0.0074 ; \mathrm{F}(1,19)=11.65$, $P=0.0029$; and $\mathrm{F}(1,19)=9.0, P=0.0074$, respectively), such that both prepregnancy obesity and gestational obesity increased the expression of MOR, but this effect was not additive (ie, high fat/high fat offspring were not different from either of the single exposure groups) (Figure 1). In the NAc, there was a main effect for both embryo and gestation, such that exposure to obesity, either prepregnancy or during gestation, increased the expression of MOR in the NAc. Effects on PENK mRNA were less extensive. A notable interaction was observed in the $\operatorname{PFC}(\mathrm{F}(1,17)=11.12$, $P=0.0039$ ), such that obesity both before and during pregnancy increased the expression of PENK, however, the combination of the two appeared to normalize expression somewhat, although not to the level of controls. A main effect for gestational exposure was also evident in the HYP $(\mathrm{F}(1,19)=11.03, P=0.0036)$, such that gestation in an obese dam led to increased PENK mRNA. And finally, DAT was evaluated in the VTA, and a main effect for gestational exposure was evident $(\mathrm{F}(1,17)=15.75, P=0.001)$, such that gestation in an obese dam led to increased DAT mRNA.

In females, the results were starkly different. There were no interactions noted, however, for every gene tested in each brain region, there was a significant effect of obesity during gestation (see Table 1 and Figure 2). In a single brain region, the NAc, an effect of prepregnancy obesity was evident for both MOR and PENK mRNA. Importantly, the direction of the effect was the opposite that seen in the males (prepregnancy obesity led to a decrease, rather than an increase, in MOR and PENK in the NAc of females). Further, for DAT in the VTA, again similar to males there was a main effect for gestation, however, the effect was in the opposite direction (gestational obesity decreased DAT mRNA in the VTA in females).

Brains were also obtained from E17.5 embryos to determine whether these effects were present before birth. Embryonic brains were divided into forebrain, midbrain, and hindbrain divisions. In the males, an interaction was noted for MOR in the hindbrain, such that an effect of prepregnancy obesity was evident, but only if the embryo 
Females
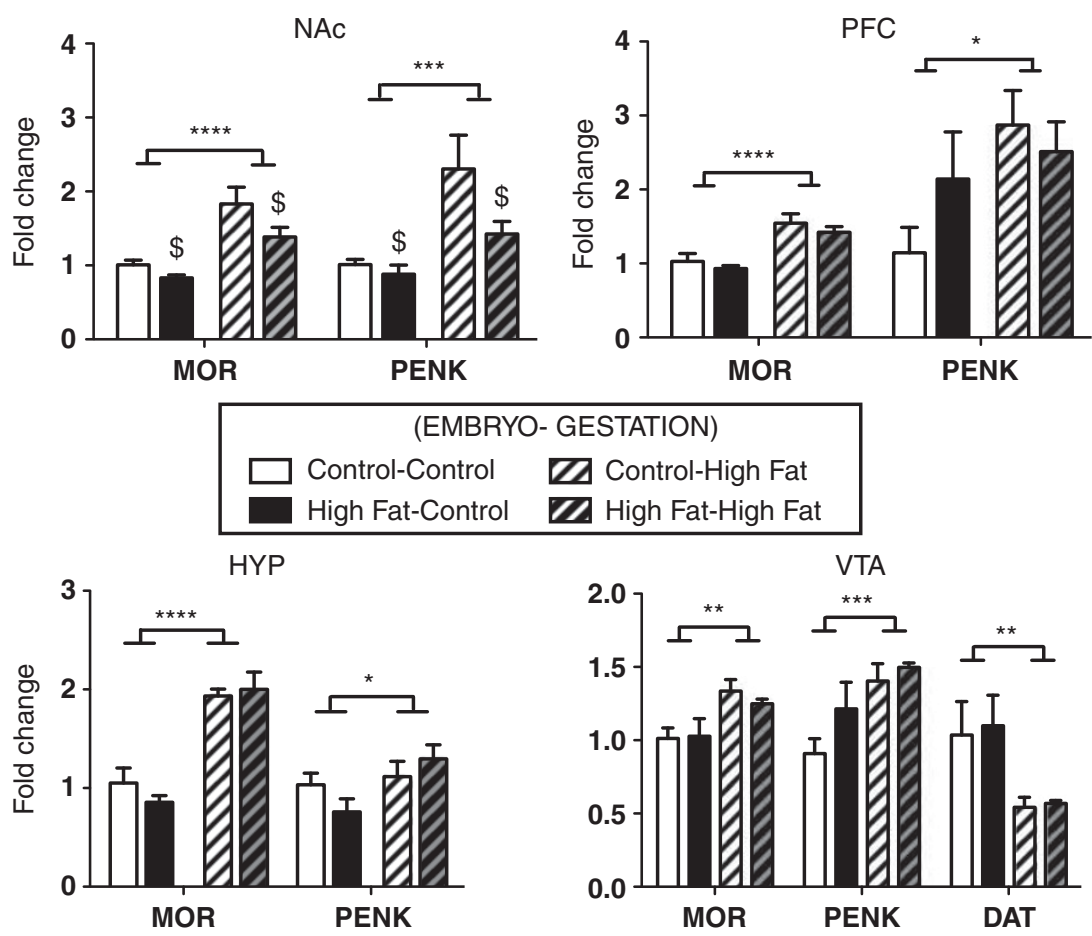

Figure 2 Gene expression in the adult female brain. mRNA for MOR and PENK was evaluated in the NAc, PFC, HYP, and VTA. DAT was also measured in the VTA. ${ }^{\#} P<0.05$, a main effect for prepregnancy obesity. Brackets indicate main effect for gestational obesity $(* P<0.05$, $* * P<0.005$, $* * * *<0.001$, ****** $P<0.000$ I, $\left.{ }^{\$} P<0.05\right)$.

was carried by a control dam $(\mathrm{F}(1,16)=6.11, P=0.025)$ (Supplementary Table S1; Figure 3). Main effects for gestation were evident in both MOR and PENK in both the midbrain and the forebrain; however in the forebrain, the effect was a decrease in expression of MOR and PENK, as opposed to increased expression seen in the adult brain. Additionally, a main effect for prepregnancy obesity was evident in PENK in the forebrain $(\mathrm{F}(1,16)=9.5, P=0.007)$. Again, in females, the effects were more limited, with significant differences observed only in the hindbrain (Supplementary Table S2; Figure 3). Main effects for gestation were noted in both MOR and PENK, however, the fold change values were very small.

DNA methylation within the promoter region of MOR was evaluated in male PFC (Figure 4), because we have previously shown that maternal HFD during pregnancy and lactation decreases MOR promoter methylation in line with increased MOR mRNA expression (Vucetic et al, 2010). Two-way ANOVA revealed a significant interaction between prepregnancy and gestation $(\mathrm{F}(1,16)=14.0, P=0.001)$, and post hoc analyses indicated a significant reduction in PFC MOR promoter methylation in the control embryos gestated by an obese dam $(P<0.03)$. A similar, but non-significant trend $(P=0.06)$ was seen in the animals exposed only to prepregnancy obesity. These decreases in MOR promoter methylation paralleled the increased mRNA expression observed in these animals. Surprisingly, offspring exposed to obesity both prepregnancy and during gestation did not differ from controls. In females, there were no differences in promoter methylation observed in the PFC, potentially due to increased variance observed across the groups.

\section{DISCUSSION}

This report is the first to demonstrate that obesity before pregnancy can affect molecular end points in the offspring brain (gene expression within the central opioid system), and thereby significantly broadens the timeframe during which maternal obesity can directly affect offspring brain development. Use of the embryo transfer design allowed for the isolation of the effect of obesity before pregnancy $v s$ the effect of obesity during pregnancy, developmental windows that are typically confounded in human and most animal studies. As predicted and consistent with previous reports in the literature, obesity during pregnancy also had a significant effect on the development of the CNS opioid system in both males and females. Notably, all offspring exposed to the HF diet, either before or during gestation, were growth restricted in utero, and showed evidence of placental dysmorphology (Sasson et al, submitted), suggesting that the observed CNS gene expression changes may be subsequent to growth restriction driven by placental dysfunction. In no instance was there an isolated effect of obesity before pregnancy (eg, without a similar result occurring in response to obesity during pregnancy), however, the converse was not true, as there were instances of a gestational effect of obesity that was not observed in response to pregestational obesity.

In males, in all brain regions, prepregnancy obesity led to an increase in MOR expression. The MOR has a critical role in the control of food intake, particularly with regard to palatable foods. Pioneering work done by Kelley and colleagues established the importance of cortico-striatal 


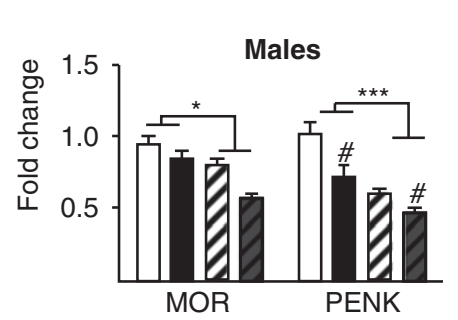

Forebrain
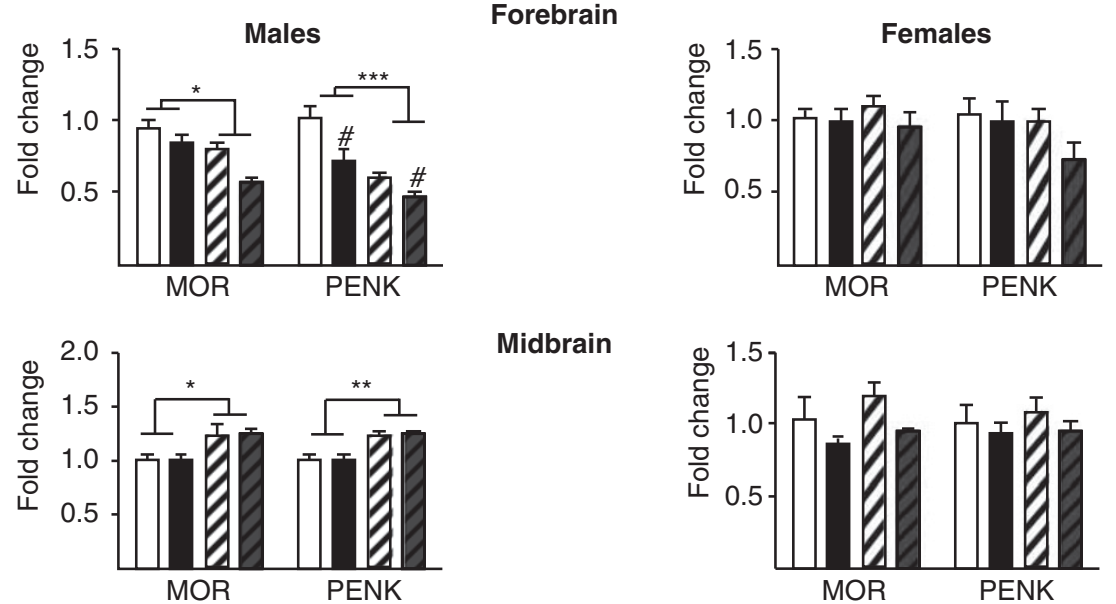

Midbrain
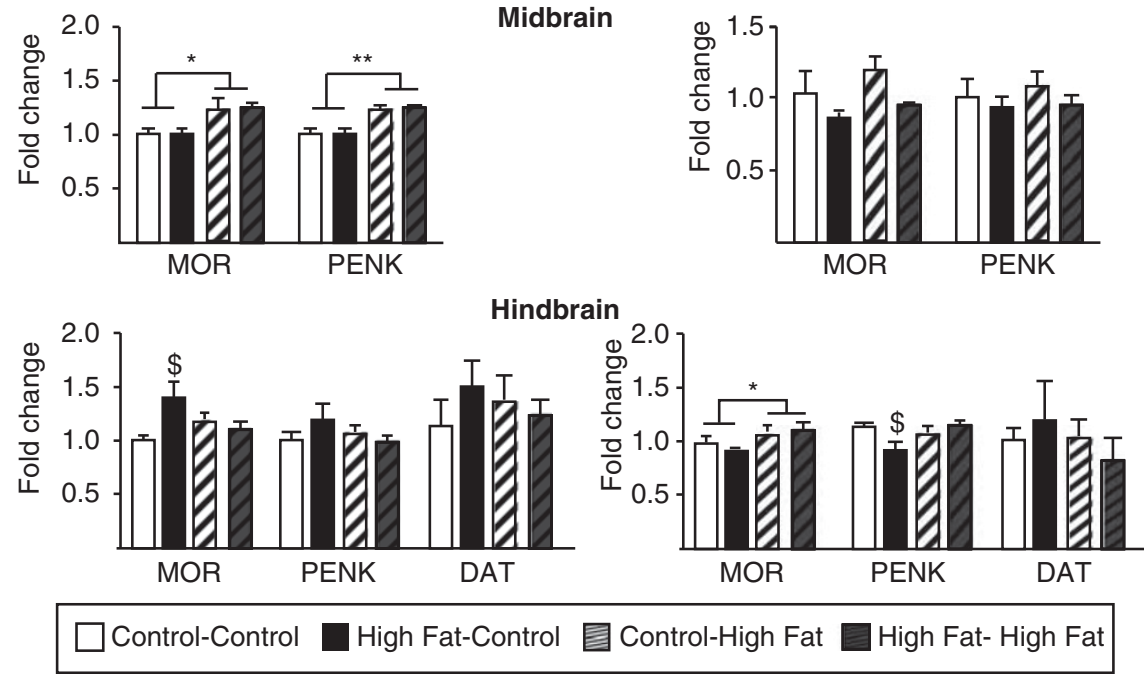

Figure 3 Gene expression in the male and female brain at E 17.5. mRNA for MOR and PENK was evaluated in the forebrain, midbrain, and hindbrain. DAT was also measured in the hindbrain. Data from both males (left) and females (right). ${ }^{\#} P<0.01$, a main effect for prepregnancy obesity in male PENK forebrain. Brackets indicate main effect for gestational obesity $(* P<0.05$, $* * P<0.0$ I, $* * * P<0.000$ I), whereas $\$(P<0.05)$ indicates a significant interaction.

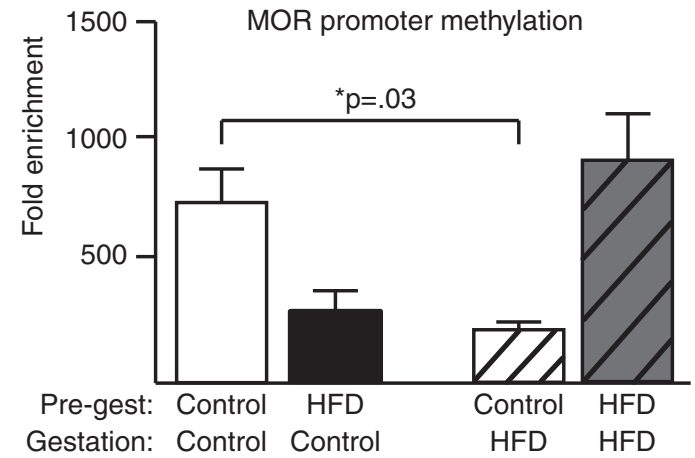

Figure 4 DNA methylation of the MOR promoter. DNA methylation was measured in the MOR promoter in samples from the male PFC. Gestation in an obese dam decreased MOR promoter methylation, consistent with an increase in mRNA expression. $* P=0.03$.

opioids in the regulation of palatable food intake (Kelley et al, 2005; Mena et al, 2011). Recently, it has been shown that an inverse agonist for the MOR can decrease the intake of palatable food, decrease sucrose preference, and decrease motivated responding for palatable foods (Ignar et al, 2011). Indeed, MOR is a target for a potential therapeutic directed toward obesity and disordered eating (Nathan et al, 2012; Ziauddeen et al, 2012). Therefore, increased signaling through the MOR may promote obesity through the selective enhancement of consumption of highly palatable, energy dense (high fat and high carbohydrate) foods, particularly in a context of food choice. In addition to the effect of prepregnancy obesity, a strong and consistent effect of obesity during pregnancy emerged, and in this case, both males and females were equally affected. Collectively, these data show that maternal obesity/consumption of an HFD has the potential to negatively impact CNS reward system circuitry development both before and during pregnancy. The implications for adverse effects on behavior extend beyond dysregulation of hedonically driven feeding, to include a broad range of behaviors in which MOR is important, such as binge-eating behavior (Nathan and Bullmore, 2009), impulsivity (Kieres et al, 2004), and the potential for altered responses to drugs of abuse, such as cocaine and heroin (Giuliano et al, 2013) as well as craving during abstinence (Theberge et al, 2012).

Enkephalin (from the precursor PENK) is the endogenous ligand for the MOR. As with MOR, prepregnancy obesity led to an increased expression of PENK in the male PFC. Gestational exposure to obesity was clearly a more potent stimulus however, as PENK expression was increased in the male HYP and PFC, as well as in all regions of the female brain when offspring were exposed to obesity during pregnancy. Previously, it has been reported that animals exposed to an HFD from E6 to PN15 displayed increased PENK in HYP (Chang et al, 2008). Additionally, rats that are naturally prone to overconsume an HFD also demonstrate increased hypothalamic expression of PENK (Chang et al, 2010). These data build on these findings by demonstrating that the effects of perinatal obesity extend beyond the hypothalamus, and that vulnerability to the effects of obesity and/or HFD begin in some cases before gestation. 
Because PENK is the endogenous ligand for the MOR, increased expression of the ligand will likely have similar functional implications as increased expression of the receptor (eg, driving an increased preference for palatable, energy dense, high-fat, and high-sugar foods).

The data from the embryonic brains further focused on the developmental critical period. It is important to consider differences between rodent and human brain development, which is well established to differ across species (Clancy et al, 2007). Cortical development events occurring from E15 term in the mouse are roughly equivalent to events that occur during the late first trimester of human pregnancy (eg, neuronal migration in the cortex, proliferation of neural progenitor/stem cells, gliogenesis, and axonogenesis). In all cases, genes that were altered in the embryonic brain were still altered in the adult brain. Because expression changes were observed in this early developmental period, the importance of the gestational period (in the absence of continued exposure to HFD/ obesity during lactation) is supported. Additionally, significant brain development occurs postnatally in the rodent (eg, postnatal days 3-11 are thought to mirror the third trimester of a human pregnancy; Livy et al, 2003), which is why HFD exposure was continued through lactation. In the present experiments, it is not possible to disentangle the effects of gestational HFD from those that occurred during lactation. So, while the embryonic period clearly represents a period of vulnerability to HFD exposure, lactation is an important developmental period as well, as alterations in nutritional status (through manipulation of litter size) have been shown repeatedly to affect brain development end points relating to appetite regulation and stress physiology (Rajia et al, 2010; Spencer and Tilbrook, 2009). Additionally, gene expression changes seen in the adult NAc that were driven by prepregnancy obesity were not apparent in the embryonic samples. Thus, the effect of embryo exposure to maternal obesity on the development of this brain region was only revealed by further unknown developmental processes occurring after birth (eg, lactation or puberty). This underscores the fact that the true impact of a periconceptional or gestational manipulation on neuronal function may lie dormant until triggered by subsequent changes in the environment or internal milieu weeks or months later. Also, it should be noted that the embryonic data cannot be directly compared with the adult data, as the size of the embryonic brain limited specificity of the dissections, and this may in part explain the differences in the direction of the changes noted in some genes between the embryonic and adult samples (eg, a different quantity of MOR + cells in the forebrain embryonic sample $v s$ the much more precise NAc dissection in the adult brain).

The observed sex differences (females less affected by prepregnancy obesity and instances of effects in the opposite direction) were not wholly unexpected, as the effects of perinatal insults are known to vary by sex (Whitaker et al, 2012). It was particularly notable that in the only region affected by prenatal obesity in the females, the NAc, gene expression changes were in the opposite direction to those seen in the males. It will be important to determine in future studies whether this differential response translates into a potentially more resilient behavioral phenotype in female offspring. Further, these changes were not apparent in the embryonic samples, which suggests a role for differences that emerge in later development (eg, sex steroids). Sex differences in DAT expression and function are well known in the literature, with females demonstrating increased DAT expression (Morissette and Paolo, 1993) and function (Bhatt and Dluzen, 2005), as well as increased dopamine release and uptake in the caudate (Walker et al, 2000). Recently, it has been shown that resveratrol induces an upregulation of DAT in female, but not in male, mice, through activation of the estrogen receptor (Liberto et al, 2012). The present findings identify an additional sex difference in DAT regulation, which may have profound implications for dopamine function in the offspring, particularly as the interplay between dopamine and opioids is a key component in the response to drugs of abuse (Nestler, 2005). Further, sex differences in opioid expression and action are well known (Dahan et al, 2008), and these data demonstrate that these sex differences can arise due to differential responses to the nutritional environment, either before or during gestation.

A limitation of the current studies is the focus on mRNA expression. An extensive literature in neuropharmacology supports the role of the MOR in reward-related behaviors. Importantly, a number of studies have also shown that changes in MOR mRNA have a direct functional consequence. A recent report demonstrated that differences in MOR mRNA across different brain regions were tightly paralleled by changes in MOR binding (Inoue et al, 2013). There are also numerous studies linking changes in MOR mRNA directly with behavioral changes, particularly as it pertains to feeding behaviors. For example, Barnes et al (2008) showed that food deprivation led to increased intake of HFD that was paralleled by an increase in MOR mRNA in the VMH/ARC. Importantly, this behavioral response (hyperphagia) was blocked by the administration of an MOR antagonist. In a study closely related to the current experiments, maternal HFD consumption during gestation was shown to increase the expression of MOR mRNA as well as an increase in preference for HFD in the offspring (a finding that has also been demonstrated convincingly in rats; Ong et al, 2013). When methyl donor supplementation was added to the diets of the pregnant dams, both the HFDinduced increase in MOR mRNA in the offspring and the increased HFD preference were blocked (Carlin et al, 2013). The link between changes in MOR mRNA and behavioral changes extends beyond the regulation of food intake. The development of allodynia following sciatic nerve damage is accompanied by decreased expression of the MOR, and this behavioral response can be reversed through administration of MOR agonist (Hervera et al, 2011). Further, sensitivity to morphine (an MOR agonist) parallels changes in MOR mRNA seen across the circadian cycle, as well as in response to differing levels of endogenous glucocorticoids (Yoshida et al, 2006).

DNA methylation of the MOR promoter was examined. Replicating our previous finding (Vucetic et al, 2010), gestational HFD decreased MOR promoter methylation in the male offspring. A similar, non-significant trend $(P=0.06)$ was observed in the animals exposed to prepregnancy obesity alone. These findings suggest that 
MOR may be susceptible to epigenetic regulation before gestation, however additional experiments would be required to directly evaluate that potential. Interestingly, the combination of prepregnancy and gestational obesity did not alter MOR promoter methylation. This pattern is consistent with other instances where the embryo derived from an obese dam then gestated in an obese environment showed a normalized phenotype (eg, PENK in male PFC and female NAc), which may be an example of predictive adaptive response (PAR) (Ikenasio-Thorpe et al, 2007). The theory of PAR typically refers to an adaptive advantage that arises when the in utero environment in which a fetus develops matches the eventual adult environment in which the animal will breed (Gluckman et al, 2005). PAR can also be used to understand developmental plasticity, in this case, specific trajectories of brain development in response to altered maternal diet before and during gestation. Here, the theory of PAR would posit that a mismatch between the nutritional environment before gestation and the environment during gestation would lead to a negative outcome/ phenotype; while conversely, when the environments are 'matched', the outcome will lead to optimal development. While in general, we did not observe a significant empirical support for PAR in our animals with these particular end points, the noted exceptions (PENK mRNA and MOR promoter methylation) may represent specific examples in which the 'matching' of the environments had a role in normalizing the adverse phenotype. PAR is typically applied in the context of prenatal undernutrition (Forrester et al, 2012), and the present limited examples suggest that the importance of environmental 'matching' may have beneficial results in the context of maternal overnutrition as well.

Collectively, these findings identify obesity, both before and during gestation, as independent maternal conditions with the potential to dramatically alter offspring brain development, specifically opioid expression within reward neural circuitry. These molecular changes are likely to contribute to the increased risk for obesity observed in offspring born to obese mothers. These findings conclusively show that obesity before pregnancy is sufficient to alter offspring brain development, and reveal notable sex differences in the response of the offspring to maternal obesity. Given that $20-38 \%$ of pregnant women in the United States are obese (Yogev and Catalano, 2009), and between 50 and $75 \%$ of women exceed the recommendation for weight gain during pregnancy (Brawarsky et al, 2005; Oken et al, 2007), the impact of these findings are widereaching and involve the majority of babies born in the United States.

\section{FUNDING AND DISCLOSURE}

The authors declare no conflict of interest.

\section{ACKNOWLEDGEMENTS}

This work was supported by NIH grants \#DK062965 (RAS) and MH087978 (TMR).

\section{REFERENCES}

Alati R, Najman JM, O'Callaghan M, Bor W, Williams GM, Clavarino A (2009). Fetal growth and behaviour problems in early adolescence: findings from the Mater University Study of Pregnancy. Int J Epidemiol 38: 1390-1400.

Barnes MJ, Primeaux SD, Bray GA (2008). Food deprivation increases the mRNA expression of micro-opioid receptors in the ventral medial hypothalamus and arcuate nucleus. Am J Physiol Regul Integr Comp Physiol 295: R1385-R1390.

Bayol SA, Farrington SJ, Stickland NC (2007). A maternal 'junk food' diet in pregnancy and lactation promotes an exacerbated taste for 'junk food' and a greater propensity for obesity in rat offspring. Br J Nutr 98: 843-851.

Berrendero F, Robledo P, Trigo JM, Martín-García E, Maldonado R (2010). Neurobiological mechanisms involved in nicotine dependence and reward: Participation of the endogenous opioid system. Neurosci Biobehav Rev 35: 220-231.

Bhatt SD, Dluzen DE (2005). Dopamine transporter function differences between male and female CD-1 mice. Brain Res 1035: 188-195.

Bilbo SD, Tsang V (2010). Enduring consequences of maternal obesity for brain inflammation and behavior of offspring. FASEB J 24: 2104-2115.

Brawarsky P, Stotland NE, Jackson RA, Fuentes-Afflick E, Escobar GJ, Rubashkin $\mathrm{N}$ et al (2005). Pre-pregnancy and pregnancy-related factors and the risk of excessive or inadequate gestational weight gain. Int J Gynaecol Obstet 91: 125-131.

Carlin J, George R, Reyes TM (2013). Methyl donor supplementation blocks the adverse effects of maternal high fat diet on offspring physiology. PLoS ONE 85: e63549.

Champlin AK, Dorr DL, Gates AH (1973). Determining the stage of the estrous cycle in the mouse by the appearance of the vagina. Biol Reprod 8: 491-494.

Chang GQ, Gaysinskaya V, Karatayev O, Leibowitz SF (2008). Maternal high-fat diet and fetal programming: increased proliferation of hypothalamic peptide-producing neurons that increase risk for overeating and obesity. J Neurosci 28: 1210712119.

Chang GQ, Karatayev O, Barson JR, Chang SY, Leibowitz SF (2010). Increased enkephalin in brain of rats prone to overconsuming a fat-rich diet. Physiol Behav 101: 360-369.

Clancy B, Kersh B, Hyde J, Darlington RB, Anand KJ, Finlay BL (2007). Web-based method for translating neurodevelopment from laboratory species to humans. Neuroinformatics 5: 79-94.

Dahan A, Kest B, Waxman AR, Sarton E (2008). Sex-specific responses to opiates: animal and human studies. Anesth Analg 107: 83-95.

Dodds L, Fell DB, Shea S, Armson BA, Allen AC, Bryson S (2011). The role of prenatal, obstetric and neonatal factors in the development of autism. J Autism Dev Disord 41: 891-902.

Forrester TE, Badaloo AV, Boyne MS, Osmond C, Thompson D, Green C et al (2012). Prenatal factors contribute to the emergence of kwashiorkor or marasmus in severe undernutrition: evidence for the predictive adaptation model. PLOS ONE 7: e35907.

Giuliano C, Robbins TW, Wille DR, Bullmore ET, Everitt BJ (2013). Attenuation of cocaine and heroin seeking by $\mu$-opioid receptor antagonism. Psychopharmacology (Berl) 227: 137-147.

Gluckman PD, Hanson MA, Spencer HG (2005). Predictive adaptive responses and human evolution. Trends Ecol Evol 20: 527-533.

Gupta A, Srinivasan M, Thamadilok S, Patel MS (2009). Hypothalamic alterations in fetuses of high fat diet-fed obese female rats. J Endocrinol 200: 239-300.

Haghighi A, Melka MG, Bernard M, Abrahamowicz M, Leonard GT, Richer L et al (2013). Opioid receptor mu 1 gene, 
fat intake and obesity in adolescence. Mol Psychiatry doi:10.1038/mp.2012.179 (E-pub ahead of print).

Hervera A, Negrete R, Leánez S, Martín-Campos JM, Pol O (2011). Peripheral effects of morphine and expression of $\mu$-opioid receptors in the dorsal root ganglia during neuropathic pain: nitric oxide signaling. Mol Pain 7: 25.

Hinkle SN, Schieve LA, Stein AD, Swan DW, Ramakrishnan U, Sharma AJ (2012). Associations between maternal prepregnancy body mass index and child neurodevelopment at 2 years of age. Int J Obes (Lond) 36: 1312-1319.

Ignar DM, Goetz AS, Noble KN, Carballo LH, Stroup AE, Fisher JC et al (2011). Regulation of ingestive behaviors in the rat by GSK1521498, a novel micro-opioid receptor-selective inverse agonist. J Pharmacol Exp Ther 339: 24-34.

Ikenasio-Thorpe BA, Breier BH, Vickers MH, Fraser M (2007). Prenatal influences on susceptibility to diet-induced obesity are mediated by altered neuroendocrine gene expression. J Endocrinol 193: 31-37.

Inoue K, Burkett JP, Young LJ (2013). Neuroanatomical distribution of $\mu$-opioid receptor mRNA and binding in monogamous prairie voles (Microtus ochrogaster) and non-monogamous meadow voles (Microtus pennsylvanicus). Neuroscience 244: 122-133.

Kelley AE, Baldo BA, Pratt WE, Will MJ (2005). Corticostriatalhypothalamic circuitry and food motivation: integration of energy, action and reward. Physiol Behav 86: 773-795.

Kieres AK, Hausknecht KA, Farrar AM, Acheson A, Hd Wit, Richards JB (2004). Effects of morphine and naltrexone on impulsive decision making in rats. Psychopharmacology (Berl) 173: $167-174$.

Klein G, Rossi GC, Waxman AR, Arout C, Juni A, Inturrisi CE et al (2009). The contribution of MOR-1 exons 1-4 to morphine and heroin analgesia and dependence. Neurosci Lett 457: 115-119.

Krakowiak P, Walker CK, Bremer AA, Baker AS, Ozonoff S, Hansen RL et al (2012). Maternal metabolic conditions and risk for autism and other neurodevelopmental disorders. Pediatrics 129: e1121-e1128.

Liberto VD, Mäkelä J, Korhonen L, Olivieri M, Tselykh T, Mälkiä A et al (2012). Involvement of estrogen receptors in the resveratrol-mediated increase in dopamine transporter in human dopaminergic neurons and in striatum of female mice. Neuropharmacology 62: 1011-1018.

Lieshout RJV, Taylor VH, Boyle MH (2011). Pre-pregnancy and pregnancy obesity and neurodevelopmental outcomes in offspring: a systematic review. Obes Rev 12: e548-e559.

Livy DJ, Miller EK, Maier SE, West JR (2003). Fetal alcohol exposure and temporal vulnerability: effects of binge-like alcohol exposure on the developing rat hippocampus. Neurotoxicol Teratol 25: 447-458.

Mena JD, Sadeghian K, Baldo BA (2011). Induction of hyperphagia and carbohydrate intake by $\mu$-opioid receptor stimulation in circumscribed regions of frontal cortex. J Neurosci 31: 3249-3260.

Morissette M, Paolo TD (1993). Sex and estrous cycle variations of rat striatal dopamine uptake sites. Neuroendocrinology 58: $16-22$.

Naef L, Moquin L, Bo GD, Giros B, Gratton A, Walker CD (2011). Maternal high-fat intake alters presynaptic regulation of dopamine in the nucleus accumbens and increases motivation for fat rewards in the offspring. Neuroscience 176: 225-236.

Nagy A, Gertsenstein M, Vintersten K, Behringer R (2003). Manipulating the Mouse Embryo: A Laboratory Manual. Cold Spring Harbor Laboratory Press: Cold Spring Harbor: NY.

Nathan PJ, Bullmore ET (2009). From taste hedonics to motivational drive: central $\mu$-opioid receptors and binge-eating behaviour. Int J Neuropsychopharmacol 12: 995-1008.

Nathan PJ, O'Neill BV, Bush MA, Koch A, Tao WX, Maltby K et al (2012). Opioid receptor modulation of hedonic taste preference and food intake: a single-dose safety, pharmacokinetic, and pharmacodynamic investigation with GSK1521498, a novel \{micro\}-Opioid Receptor Inverse Agonist. J Clin Pharmacol 52: 464-474.

Nestler EJ (2005). Is there a common molecular pathway for addiction? Nat Neurosci 8: 1445-1449.

Oken E, Taveras EM, Kleinman KP, Rich-Edwards JW, Gillman MW (2007). Gestational weight gain and child adiposity at age 3 years. Am J Obstet Gynecol 196: e1-e8.

Ong ZY, Muhlhausler BS (2011). Maternal 'junk-food' feeding of rat dams alters food choices and development of the mesolimbic reward pathway in the offspring. FASEB $J$ 25: 2167-2179.

Ong ZY, Wanasuria AF, Lin MZ, Hiscock J, Muhlhausler BS (2013). Chronic intake of a cafeteria diet and subsequent abstinence. Sex-specific effects on gene expression in the mesolimbic reward system. Appetite 65: 189-199.

Pfaffl MW (2010). The ongoing evaluation of qPCR. Methods 50: 215-216.

Rajia S, Chen H, Morris MJ (2010). Maternal overnutrition impacts offspring adiposity and brain appetite markers-modulation by postweaning diet. J Neuroendocrinol 22: 905-914.

Ray GT, Croen LA, Habel LA (2009). Mothers of children diagnosed with attention-deficit/hyperactivity disorder: health conditions and medical care utilization in periods before and after birth of the child. Med Care 47: 105-114.

Rodriguez A (2010). Maternal pre-pregnancy obesity and risk for inattention and negative emotionality in children. J Child Psychol Psychiatry 51: 134-143.

Sasson IE, Vitins AP, Mainigi MA, Moley KH, Simmons RA (submitted). Pre-gestational versus gestational exposure to maternal obesity differentially programs the offspring.

Schaefer BC, Schaefer ML, Kappler JW, Marrack P, Kedl RM (2001). Observation of antigen-dependent CD8 + T-cell/dendritic cell interactions in vivo. Cell Immunol 214: 110-122.

Shalev U, Tylor A, Schuster K, Frate C, Tobin S, Woodside B (2010). Long-term physiological and behavioral effects of exposure to a highly palatable diet during the perinatal and post-weaning periods. Physiol Behav 101: 494-502.

Shin AC, Pistell PJ, Phifer CB, Berthoud HR (2010). Reversible suppression of food reward behavior by chronic mu-opioid receptor antagonism in the nucleus accumbens. Neuroscience 170: $580-588$.

Shirley B, Wortham JW Jr, Condon-Mahony M (1986). Mating and embryo yield of mice injected with gonadotropins on specific days of the estrous cycle and in acyclic periods. Exp Biol 46: $83-88$.

Spencer SJ, Tilbrook A (2009). Neonatal overfeeding alters adult anxiety and stress responsiveness. Psychoneuroendocrinology 34: 1133-1143.

Sullivan EL, Grayson B, Takahashi D, Robertson N, Maier A, Bethea CL et al (2010). Chronic consumption of a high-fat diet during pregnancy causes perturbations in the serotonergic system and increased anxiety-like behavior in nonhuman primate offspring. J Neurosci 30: 3826-3830.

Teegarden SL, Scott AN, Bale TL (2009). Early life exposure to a high fat diet promotes long-term changes in dietary preferences and central reward signaling. Neuroscience 162: 924-932.

Theberge FR, Pickens CL, Goldart E, Fanous S, Hope BT, Liu QR et al (2012). Association of time-dependent changes in $\mathrm{mu}$ opioid receptor mRNA, but not BDNF, TrkB, or MeCP2 mRNA and protein expression in the rat nucleus accumbens with incubation of heroin craving. Psychopharmacology (Berl) 224: 559-571.

Tozuka Y, Kumon M, Wada E, Onodera M, Mochizuki H, Wada K (2010). Maternal obesity impairs hippocampal BDNF production and spatial learning performance in young mouse offspring. Neurochem Int 57: 235-247. 
Vucetic Z, Kimmel J, Totoki K, Hollenbeck E, Reyes TM (2010). Maternal high-fat diet alters methylation and gene expression of dopamine and opioid-related genes. Endocrinology 151: 4756-4764.

Walker QD, Rooney MB, Wightman RM, Kuhn CM (2000). Dopamine release and uptake are greater in female than male rat striatum as measured by fast cyclic voltammetry. Neuroscience 95: 1061-1070.

Wang H, Dey SK (2006). Roadmap to embryo implantation: clues from mouse models. Nat Rev Genet 7: 185-199.

Whitaker KW, Totoki K, Reyes TM (2012). Metabolic adaptations to early life protein restriction differ by offspring sex and postweaning diet in the mouse. Nutr Metab Cardiovasc Dis 22: 1067-1074.

Yogev Y, Catalano PM (2009). Pregnancy and obesity. Obstet Gynecol Clin North Am 36: 285-300.
Yoshida M, Kiyofuji H, Koyanagi S, Matsuo A, Fujioka T, To $\mathrm{H}$ et al (2006). Glucocorticoid is involved in food-entrainable rhythm of mu-opioid receptor expression in mouse brainstem and analgesic effect of morphine. J Pharmacol Sci 101: 77-84.

Yu ZB, Han SP, Zhu GZ, Zhu C, Wang XJ, Cao XG et al (2011). Birth weight and subsequent risk of obesity: a systematic review and meta-analysis. Obes Rev 12: 525-542.

Zhang M, Kelley AE (2000). Enhanced intake of high-fat food following striatal mu-opioid stimulation: microinjection mapping and fos expression. Neuroscience 99: 267-277.

Ziauddeen H, Chamberlain SR, Nathan PJ, Koch A, Maltby K, Bush $\mathrm{M}$ et al (2012). Effects of the mu-opioid receptor antagonist GSK1521498 on hedonic and consummatory eating behaviour: a proof of mechanism study in binge-eating obese subjects. $\mathrm{Mol}$ Psychiatry doi:10.1038/mp.2012.154 (E-pub ahead of print).

Supplementary Information accompanies the paper on the Neuropsychopharmacology website (http://www.nature.com/npp) 